\title{
Comparison of L-Selectin and CD11b on Neutrophils of Adults and Neonates during the First Month of Life
}

\author{
SHELLY K. KIM, SUSAN E. KEENEY, SCOTT K. ALPARD, AND FRANK C. SCHMALSTIEG \\ Departments of Pediatrics, Human Biological Chemistry and Genetics, [F.C.S.] and Surgery [S.K.K., \\ S.E.K., S.K.A.], The University of Texas Medical Branch, Galveston, Texas, U.S.A.
}

\begin{abstract}
ABSTR
The newborn infant is particularly susceptible to infection in
the first weeks of life and this may be, in part, related to
functional impairment of neonatal neutrophils in regard to ad-
herence, chemotaxis, and migration. Differences in expression of
the neutrophil adherence molecules, L-selectin and CD11b/CD18
(Mac-1), have been previously demonstrated in cord blood and in
very young infants ( $\leq 48$ h) compared with adults, but it is
unknown for how long these differences persist. We measured
surface expression of neutrophil L-selectin and CD11b using flow
cytometry in healthy term human newborns from $24 \mathrm{~h}$ through 4
wk of age. We also measured levels of soluble L-selectin using an
ELISA in neonates though the age of 4 wk. Compared with
adults, neonates expressed significantly less L-selectin on resting
neutrophils through 4 wk of age, with the lowest levels being at
24 h. Expression of L-selectin on the neutrophil after activation
with $N$-formyl-methionyl-leucyl-phenylalanine was less in the
neonate than in the adult $(p<0.05)$ for the first week of life.
Soluble L-selectin showed a steady increase with age in the
\end{abstract}
The newborn infant is particularly susceptible to severe bacterial and fungal infections $(1,2)$. It is unknown for how long this susceptibility persists, but it is a clinical practice to treat these infants as immunocompromised for at least the length of the neonatal period (first $28 \mathrm{~d}$ of life). Neonatal neutrophils, compared with those of adults, have been found to have functional impairments in their ability to adhere to an activated endothelial surface and to migrate in response to chemotaxins (2-6). These impairments may delay or prevent neutrophils from reaching inflammatory sites, thus inhibiting the ability of the neonate to effectively fight infection.

Received October 10, 2001; accepted July 30, 2002.

Address correspondence to: Susan E. Keeney, M.D., Division of Neonatology, Rt. 0526, University of Texas Medical Branch, Galveston, TX 77555-0526, U.S.A.; e-mail: skeeney@utmb.edu

Supported by a Child Health Research Center Grant (HD 27841 Center Grant) from the National Institutes of Child Health and Human Development.

Current address for S.K.K.: 13601 Orchard Court, Gregory, MI 48137, U.S.A.

DOI: 10.1203/01.PDR.0000041515.78650.11 neonates. Soluble L-selectin was significantly lower in the 24-h neonate compared with the adult and was higher in the 4-wk neonate compared with the adult. CD11b expression was similar between neonates and adults on unstimulated neutrophils, but upon activation, the neonatal neutrophil demonstrated significantly lower up-regulation of CD11b on the neutrophil surface through 4 wk of age compared with adults $(p<0.05$ for all ages except $1 \mathrm{wk}$ ). These findings that differences in expression of L-selectin and CD11b from that in adults persist throughout the neonatal period provide further evidence that these differences may play a role in the neutrophil defects observed during the neonatal period. (Pediatr Res 53: 132-136, 2003)

\section{Abbreviations}

FMLP, N-formyl-methionyl-leucyl-phenylalanine

Mac-1 (CD11b/CD18), macrophage antigen-1

PMN, polymorphonuclear leukocyte
Adherence of neutrophils to the vascular endothelium is an early step in the process of neutrophil migration into inflamed tissue and is mediated by proteins expressed on the surface of the neutrophil and the endothelium. Members of two important families of adhesion molecules located on the surface of the neutrophil are L-selectin and the $\beta 2$-integrins. L-Selectin is found on neutrophils and other leukocytes and recognizes carbohydrate ligands on the endothelial cell, including sialyl Lewis $\chi(7)$. L-Selectin is thought to mediate, along with other selectin proteins, initial attachment of neutrophils to the endothelium and "rolling" down the vessel under conditions of shear force (7). L-Selectin is constitutively expressed on the neutrophil surface and is rapidly down-regulated within minutes of in vitro neutrophil activation $(8,9)$. Down-regulation of L-selectin appears to be due to proteolytic cleavage of the protein from the neutrophil surface, leaving a functionally intact soluble extracellular portion of the molecule (10).

The $\beta 2$ leukocyte integrins, lymphocyte function-associated antigen (LFA-1), Mac-1, and p150,95, are adhesion glycoproteins expressed on the leukocyte surface that are involved in 
neutrophil adherence $(11,12)$. CD11b (Mac-1) is expressed constitutively at low levels on the neutrophil surface. It is stored in intracellular granules and translocated to the neutrophil surface upon activation of the neutrophil, resulting in an increase in surface expression $(12,13)$. Not only do neutrophils increase their number of surface receptors, but the affinity of CD11b receptors increases after stimulation (14). CD11b promotes firm attachment of the neutrophil to the endothelium, which then allows transendothelial migration into inflamed tissue (11).

Previous studies have primarily examined the expression of L-selectin and CD11b on neutrophils from human cord blood. Cord blood neutrophils express a decreased amount of Lselectin when compared with adult controls $(4,5,15,16)$. Most studies have shown a diminished up-regulation of CD11b expression after stimulation of neonatal neutrophils $(5,17-19)$. These studies suggest that there are early differences in expression of the adhesion molecules on neutrophils of cord blood and in very young term neonates. However, no studies have systematically compared neonatal expression with that of the adult in term neonates older than $48 \mathrm{~h}$, nor have they examined when expression of adhesion molecules approximates that of the adult. Because the deficit in neonatal neutrophil function probably lasts for several weeks, we hypothesized that differences in adhesion molecule expression between adults and neonates would persist throughout the neonatal period. To study the expression of the adhesion molecules over the neonatal period, we examined the expression of $\mathrm{L}$-selectin and $\mathrm{CD} 11 \mathrm{~b}$ on activated and unactivated neutrophils of term neonates at 24 and $72 \mathrm{~h}$ and 1,2, and 4 wk of life. We also examined amounts of soluble L-selectin in the serum of neonates through $4 \mathrm{wk}$ of life.

\section{MATERIALS AND METHODS}

Selection of research subjects. The Institutional Review Board of The University of Texas Medical Branch approved the protocol for use of human subjects in this study. Informed consent was obtained from a parent of each participant before inclusion. Peripheral blood was obtained by venipuncture from 38 full-term neonates at the following ages: $24 \mathrm{~h}(n=12), 72 \mathrm{~h}(n=9), 7 \mathrm{~d}(n=7), 2 \mathrm{wk}(n=$ $7)$, and $4 \operatorname{wk}(n=8)$. Some infants had blood drawn more than once. Not all measurements were completed for each infant due to occasional inadequate sample size. The figure legends give the numbers of infants in each specific data set. Infants were excluded who were preterm $(\leq 36$ wk gestation), had documented infection with positive blood cultures or suspected infection requiring antibiotic treatment, had 5 -min Apgar scores of $<8$, or had life-threatening congenital anomalies. Infants were included if they met the inclusion criteria and if they had another indication for venipuncture at one of the sampling time points. Maternal history and history of neonatal complications were obtained from the infant's medical record. Twenty-eight adult controls were included in the study. Their ages ranged from 20 to $40 \mathrm{y}$ of age and all were in good health without symptoms of infection.
Preparation of neutrophils. Peripheral blood (approximately $0.5 \mathrm{~mL}$ ) was obtained by venipuncture, placed in a heparinized tube, and immediately transported on ice to the laboratory for processing. Processing was accomplished within $1 \mathrm{~h}$ of collection. Adult samples were analyzed concurrently with neonatal blood samples and were processed in a similar fashion.

For measurement of L-selectin and CD11b on unactivated peripheral blood neutrophils, aliquots of whole blood were incubated at $4^{\circ} \mathrm{C}$ for 25 min with murine antibody to human L-selectin (Leu 8, BD Biosciences, San Jose, CA, U.S.A.) at a final concentration of $2 \mu \mathrm{g} / \mathrm{mL}$ and murine antibody to human CD11b (DAKO, Carpinteria, CA, U.S.A.) at a final concentration of $10 \mu \mathrm{g} / \mathrm{mL}$. Both primary antibodies were conjugated to R-phycoerythrin. A simultaneous phycoerythrin-conjugated isotype $\operatorname{IgG}$ control was run with each set of samples. The red blood cells were then lysed using FACS lysis buffer (BD Biosciences) and rinsed. The remaining white blood cells were fixed with $1 \%$ paraformaldehyde and stored at $4{ }^{\circ} \mathrm{C}$ in the dark until analysis by flow cytometry. After fixation, samples were stored for up to $72 \mathrm{~h}$ before flow cytometric analysis. We verified that $\mathrm{L}$-selectin and $\mathrm{CD} 11 \mathrm{~b}$ expression on unstimulated neutrophils did not differ between fixed samples analyzed immediately and those stored for up to $72 \mathrm{~h}$ before analysis.

For measurement of L-selectin and CD11b on activated peripheral blood neutrophils, $N$-formyl-methionyl-leucylphenylalanine (FMLP) was used as the activating agent. FMLP $\left(10^{-7} \mathrm{M}\right.$ final concentration) was added to aliquots of whole blood and the samples were incubated at $37^{\circ} \mathrm{C}$ for $30 \mathrm{~min}$. After incubation and rinsing with PBS, samples were then stained with L-selectin and CD11b as described above and processed in an identical manner to the unstimulated samples.

Flow cytometry. Samples were analyzed by single-color flow cytometry in a flow cytometer equipped with a $15-\mathrm{mW}$ argon-ion laser tuned to $488 \mathrm{nM}$ (FACScan, BD Biosciences). An electronic gate was set on the neutrophil and non-neutrophil populations based on forward-angle versus light scatter. Analyses were conducted with the LYSIS II program. CaliBRITE beads were run before each analysis to monitor instrument performance and to set detector levels for phycoerythrin. Mean channel fluorescence of each sample was corrected by the IgG control.

Measurement of soluble L-selectin. Samples were obtained for soluble $\mathrm{L}$-selectin from adults and neonates through $4 \mathrm{wk}$ of age. Serum samples were processed for measurement of soluble L-selectin by centrifugation of whole blood at $9700 \times g$ and freezing at $-70^{\circ} \mathrm{C}$ until measurement. Soluble $\mathrm{L}$-selectin was measured using a sandwich ELISA method (Human L-Selectin Immunoassay, R\&D Systems, Minneapolis, MN, U.S.A.). Samples were diluted at 1:100 and analyzed in duplicate. Human recombinant L-selectin controls were used at concentrations of $2.5-33 \mathrm{ng} / \mathrm{mL}$ to develop a standard curve.

Statistical analysis. Results of mean channel fluorescence were expressed as mean $\pm \mathrm{SD}$. Differences between neonates and adults at each neonatal age were analyzed by one-way ANOVA using Newman-Keuls correction for multiple comparisons. For non-normal distributions, a Kruskal-Wallis analysis was used with Mann-Whitney rank sum tests to compare 
groups. Comparisons of results by mode of delivery were also made using ANOVA. Comparisons of other neonatal complications and of leukocyte and granulocyte counts were made using unpaired $t$ tests and linear regression analysis. A difference of $p<0.05$ was considered significant.

\section{RESULTS}

Blood was obtained from 38 full-term neonates. Mean birth weight of neonates was $3481.3 \pm 449.2 \mathrm{~g}$. Fifty-two percent were male and all were inborn. The mean Apgar score at 5 min was 8.96 .

L-Selectin expression. Figure 1 demonstrates L-selectin expression on neonatal neutrophils at 24 and $72 \mathrm{~h}$ and at 1,2, and 4 wk of life compared with adult neutrophils. The mean channel fluorescence of L-selectin on neonatal neutrophils was significantly lower than that on adults from $24 \mathrm{~h}$ through $4 \mathrm{wk}$. Expression at these time points was $41-60 \%$ of that in the adult and was lowest at $24 \mathrm{~h}$.

After activation with FMLP, adult neutrophils expressed less than $50 \%$ of their baseline, unstimulated L-selectin. Activated neonatal neutrophils expressed less L-selectin than did adults through $1 \mathrm{wk}$ of age. However, the percent of L-selectin lost from each unstimulated sample of adults and neonates did not differ at any of the time points.

Soluble L-selectin. Levels of soluble L-selectin in the serum of adults and term neonates through $4 \mathrm{wk}$ of age are shown in Figure 2. Values for soluble L-selectin are in the range of those reported by previous investigators $(15,20)$. Soluble L-selectin was lowest at $24 \mathrm{~h}$ and showed an increase through the neonatal period to above adult levels at $4 \mathrm{wk}$. Soluble L-selectin was significantly lower in the 24-h neonate compared with the

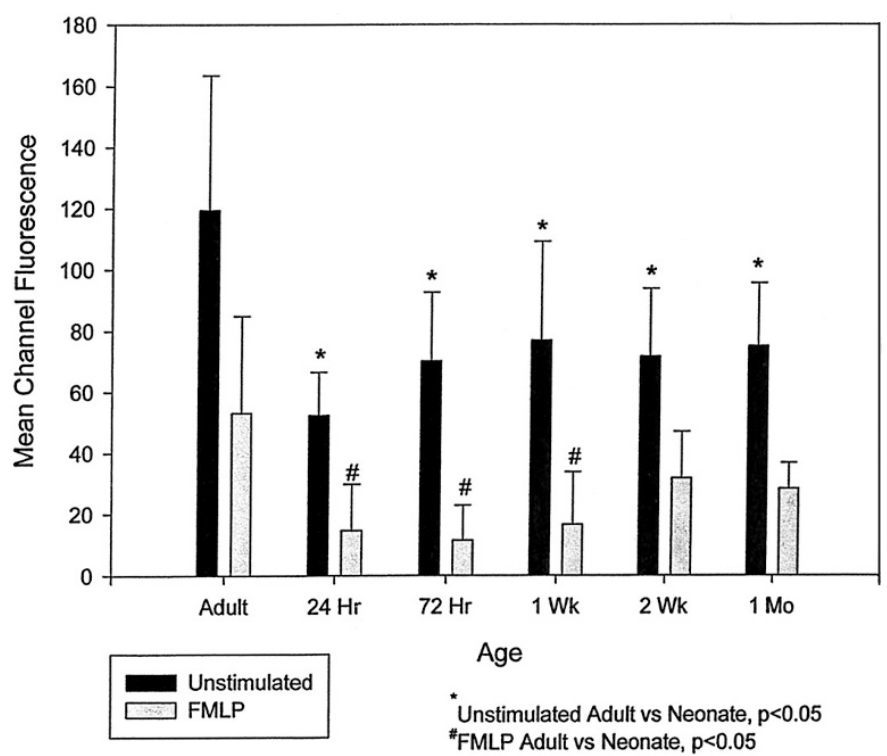

Figure 1. Mean channel fluorescence for L-selectin on unstimulated neutrophils (black bar) and neutrophils stimulated with FMLP (gray bar). Data are expressed as mean $\pm \mathrm{SD} ;{ }^{*} p<0.05$ for adult $v s$ neonate in unstimulated samples; ${ }^{*} p<0.05$ for adult $v s$ neonate in FMLP-stimulated samples. Numbers in each group are as follows: unstimulated-adult $(n=28), 24 \mathrm{~h}(n=9)$, $72 \mathrm{~h}(n=7), 1 \mathrm{wk}(n=8), 2 \mathrm{wk}(n=6)$, and $4 \mathrm{wk}(n=8)$; stimulated-adult $(n=24), 24 \mathrm{~h}(n=9), 72 \mathrm{~h}(n=6), 1 \mathrm{wk}(n=5), 2 \mathrm{wk}(n=6)$, and $4 \mathrm{wk}$ $(n=6)$.

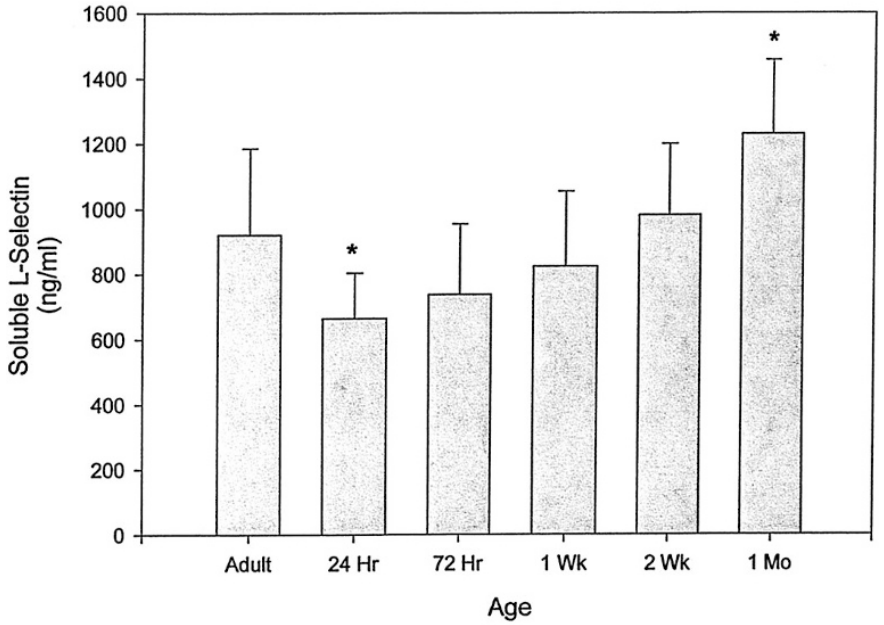

Figure 2. Soluble L-selectin $(\mathrm{ng} / \mathrm{mL})$ in serum of adults and neonates through 2 wk of age. Data are expressed as mean $\pm \mathrm{SD} ;{ }^{*} p<0.05$ for adult $v s$ neonate. Numbers in each group are as follows: adult $(n=8), 24 \mathrm{~h}(n=8), 72 \mathrm{~h}(n=$ $5), 1 \mathrm{wk}(n=5), 2 \mathrm{wk}(n=7)$, and $4 \mathrm{wk}(n=7)$.

adult. Interestingly, it was significantly higher than the adult and than all other neonates at $4 \mathrm{wk}$ of age.

CD11b expression. The expression of $\mathrm{CD} 11 \mathrm{~b}$ on peripheral blood neutrophils of adults and neonates is shown in Figure 3. There were no differences in CD11b expressed on the unstimulated neutrophils of neonates and adults. After activation of neutrophils with FMLP, the neonatal neutrophil expressed a significantly lower amount of $\mathrm{CD} 11 \mathrm{~b}$ than did the adult neutrophil through age $4 \mathrm{wk}$ except at the 1-wk time point.

Clinical correlates to adhesion molecule expression. Delivery was vaginal in $50 \%$ of infants and by cesarean section in $50 \%$. Of those with cesarean section, $39 \%$ underwent labor and $61 \%$ had elective cesarean section without labor. For those

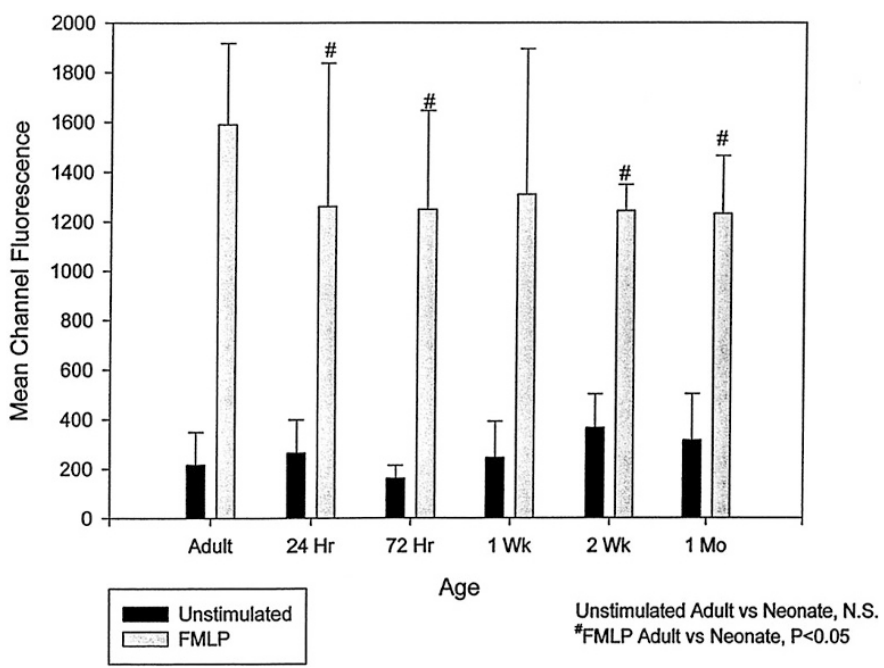

Figure 3. Mean channel fluorescence for $\mathrm{CD} 11 \mathrm{~b}$ on unstimulated neutrophils (black bar) and neutrophils stimulated with FMLP (gray bar). Data are expressed as mean $\pm \mathrm{SD} ;{ }^{*} p<0.05$ for adult $v s$ neonate in unstimulated samples; ${ }^{\#} p<0.05$ for adult $v s$ neonate in FMLP-stimulated samples. Numbers in each group are as follows: unstimulated - adult $(n=28), 24 \mathrm{~h}(n=9)$, $72 \mathrm{~h}(n=8), 1 \mathrm{wk}(n=7), 2 \mathrm{wk}(n=6)$, and $4 \mathrm{wk}(n=8)$; stimulated-adult $(n=24), 24 \mathrm{~h}(n=9), 72 \mathrm{~h}(n=6), 1 \mathrm{wk}(n=5), 2 \mathrm{wk}(n=5)$, and $4 \mathrm{wk}$ $(n=8)$. 
samples obtained within the first $3 \mathrm{~d}$ of life, L-selectin values were $53.54 \pm 13.88$ for vaginal delivery and $58.12 \pm 11.7$ for cesarean section without labor. CD11b values were $275.03 \pm$ 137.14 for vaginal delivery and $140.96 \pm 56.22$ for cesarean section without labor. There were no statistical differences between L-selectin and CD11b values by mode of delivery. There were also no differences between cesarean section with or without labor. Four infants had fetal distress with fetal heart rate decelerations requiring emergency delivery. Three of these were in the cesarean section with labor group and one was in the vaginal group. There were no differences in L-selectin or $\mathrm{CD} 11 \mathrm{~b}$ expression between these infants and the cesarean section group.

Leukocyte counts were available in nine infants. In these infants, levels of L-selectin and CD11b on the neutrophil surface did not correlate to total leukocyte or granulocyte counts (NS by linear regression analysis). Despite the exclusion criteria, some complications were noted in the histories of the subjects. These complications included ABO incompatibility $(n=6)$, nuchal cord $(n=3)$, gestational diabetes $(n=3)$, and pregnancy-induced hypertension $(n=2)$. Although the numbers were low, no differences between the mean L-selectin or $\mathrm{CD} 11 \mathrm{~b}$ values were noted in the groups with and without these complications.

\section{DISCUSSION}

In the present study, we demonstrated that expression of L-selectin is lower on human neonatal neutrophils compared with adults and that this difference persists for at least $4 \mathrm{wk}$. Our findings of lower L-selectin on unstimulated term neonatal neutrophils are similar to those of previous studies in cord blood $(4,5,15,16)$ and in neonates up to $48 \mathrm{~h}$ of age (4). Koenig et al. (21) have reported that lysates of PMN from term neonatal cord blood have lower total L-selectin than adults, corroborating a cellular deficiency in the neonate. However, our findings of a similar loss of L-selectin compared with baseline between neonatal and adult neutrophils after FMLP stimulation differ from most previous studies, which have reported less shedding of L-selectin from neonates $(4,5,21)$. We used high doses of FMLP, which may have masked differential losses at lower FMLP concentration. Our results agree with those of Rebuck et al. (15), whose method of processing neutrophils was similar to ours (processing at $4^{\circ} \mathrm{C}$ and staining of whole blood). Several investigators have demonstrated that temperature affects levels of the adhesion molecules on neutrophils, presumably by low-level activation (15, $22)$. Processing at higher temperatures $\left(30^{\circ}-37^{\circ} \mathrm{C}\right)$ and with commonly used procedures to isolate neutrophils, such as dextran sedimentation and Ficoll/Hypaque sedimentation, diminishes the ability of neutrophils to respond to FMLP (15, 22). Therefore, our method of processing neonatal neutrophils may have allowed demonstration of a greater response to FMLP.

Several mechanisms could account for the lower constitutive expression of L-selectin on the neonatal neutrophil. Smith et al. (23) reported that neutrophils from human preterm fetuses have expression of $\mathrm{L}$-selectin similar to that of the adult. This would suggest that the developing infant is capable of producing L-selectin on the surface of its neutrophils. It is possible that soluble proteases are released around the time of term gestation that degrade the L-selectin molecule or that there is a developmental change at term rendering the L-selectin molecule more susceptible to cleavage by protease. It is also possible that a cytokine or other compound is released at the time of term gestation that results in partial activation of the neutrophil and loss of L-selectin. On the other hand, our findings that neonatal neutrophils are able to lose a substantial amount of L-selectin in response to FMLP would suggest that they are not previously activated.

Regardless of mechanism, these observed adherence differences could account, at least in part, for the observed defects in neonatal neutrophil function. Evidence that $\mathrm{L}$-selectin is important for early neutrophil recognition of inflammatory sites includes findings that previously activated neutrophils, which have diminished L-selectin on their surfaces, do not localize to sites of inflammation (9). Multiple studies have shown the importance of L-selectin for certain inflammatory conditions, although some conditions appear to be L-selectin independent $(7,24)$. In regard to the neonate, Anderson et al. (4) have shown that the decrease in adhesion of cord blood neutrophils under conditions of shear force is directly related to diminished L-selectin levels on the neutrophils. In neonatal rabbits, Fortenberry et al. (25). have demonstrated decreased emigration of neutrophils into an inflammatory site that was L-selectin and CD18-dependent. Studies have shown that neonatal L-selectin expression is decreased after dexamethasone treatment (26) and in cord blood of neonates with acute bacterial infection (16).

In regard to soluble L-selectin, our finding that 24 -h neonates have lower levels than adults is comparable to results of previous studies, which have reported lower levels of soluble L-selectin in the cord blood of neonates compared with adults $(15,21,27)$. Buhrer et al. (27). found the lowest levels in cord blood of premature infants with a correlation between soluble L-selectin and gestational age. Similarly, in our neonates, there was a trend toward increases in soluble L-selectin with chronologic age. In fact, the soluble L-selectin in 1-mo neonates was higher than adults. If $\mathrm{L}$-selectin is lost from the neonatal neutrophil surface because of activation associated with delivery, soluble L-selectin would be expected to be higher in the early neonatal samples than in adults. However, soluble Lselectin may not be simply correlated with expression or loss of L-selectin on circulating neutrophils, but may be related to long-term neutrophil turnover and shedding of L-selectin (15, 20). In keeping with this hypothesis, Scheiffenbaum et al. (20) have shown that the amounts of soluble L-selectin found in human serum are 10-25 times higher than could be accounted for by release of L-selectin from the number of neutrophils present in their samples. Because neonates have diminished bone marrow stores of neutrophils (28), this deficiency may contribute to lower levels of overall neutrophil turnover and, hence, lower soluble L-selectin concentration.

We found no differences in levels of CD11b expression between unstimulated neonatal and adult neutrophils. As expected, all samples showed significantly increased expression after activation with FMLP. However, after FMLP stimulation, 
neutrophils from neonates throughout the neonatal period showed reduced up-regulation of $\mathrm{CD} 11 \mathrm{~b}$ compared with adults, except at the 1-wk time point. Our results are in accordance with other studies of $\mathrm{CD} 11 \mathrm{~b}$ up-regulation on neutrophils from cord blood $(5,17-19)$. The findings of Jones et al. (13) suggest that the mechanism of decreased neonatal up-regulation may be deficient mobilization from gelatinaserich intracellular granules. Anderson et al. (18) have shown that the diminished up-regulation of Mac-1 on neonatal (approximately $48 \mathrm{~h}$ of age) and cord blood neutrophils causes decreased adherence in response to FMLP. They also found that diminished neonatal neutrophil transendothelial migration was related, not only to quantitative abnormalities in CD11b, but also to functional deficits in stimulated binding to the ligand, ICAM-1. CD11b up-regulation has been reported in the clinical setting of neonatal sepsis $(29,30)$ and extracorporeal membrane oxygenation (31).

We found no statistical differences in adhesion molecule expression with mode of delivery. Other studies have shown no differences with mode of delivery, as well $(5,16,19,29)$. However, Weinschenk et al. (32) did find a positive correlation between $\mathrm{CD} 11 \mathrm{~b}$ expression and labor length. Usmani et al. (33) showed no differences in PMN function (chemiluminescence, motility, and chemotaxis) related to labor or mode of delivery. Interestingly, these authors (33) did note differences in PMN function after emergency cesarean section, which they attributed to the effects of decreased uteroplacental perfusion and asphyxia. We found no differences in adhesion molecule expression in the four infants in our study whose deliveries were by emergency cesarean section. However, infants whose 5 -min Apgar scores were $<8$ were excluded from the study, so we cannot comment about the effects of asphyxia.

In summary, we demonstrated that, compared with the adult, the term neonate has reduced expression of neutrophil L-selectin and diminished up-regulation of $\mathrm{CD} 11 \mathrm{~b}$ through $4 \mathrm{wk}$ of age. Although the mechanism is unknown, in view of the prolonged nature of these differences, they are probably not related to a brief physiologic response associated with delivery. The differences in expression of these adhesion molecules reflect the time course of the persistent neutrophil function defects and increased susceptibility to infection of the neonate. This is further evidence that the adherence molecules, L-selectin and CD11b, play a role in the observed neutrophil function defects of the neonate.

Acknowledgments. The authors thank Ms. Kimberley Palkowetz for technical assistance with flow cytometric analysis. We also thank Ms. Freda Purnell for assistance with completion of the manuscript and Dr. Dennis Kim for editorial and graphical assistance.

\section{REFERENCES}

1. Hill HR 1987 Biochemical, structural, and functional abnormalities of polymorphonuclear leukocytes in the neonate. Pediatr Res 22:375-382

2. Wilson CB 1986 Immunological basis for increased susceptibility of the neonate to infection. J Pediatr 108:1-12

3. Anderson DC, Hughes BJ, Smith CW 1981 Abnormal mobility of neonatal polymorphonuclear leukocytes. J Clin Invest 68:863-874

4. Anderson DC, Abbassi O, Kishimoto TK, Koenig JM, McIntire LV, Smith CW 1991 Diminished lectin-, epidermal growth factor-, complement binding domain-cell ad- hesion molecule-1 on neonatal neutrophils underlies their impaired CD18independent adhesion to endothelial cells in vitro. J Immunol 146:3372-3379

5. Torok C, Lunkahl J, Hed J, Lagercrantz H 1991 Diversity in regulation of adhesion molecules (Mac-1 and L-selectin) in monocytes and neutrophils from neonates and adults. Arch Dis Child 68:561-565

6. Klein RB, Fischer TJ, Gar SE, Biberstein M, Rich KC, Stiehm ER 1997 Decreased mononuclear and polymorphonuclear chemotaxis in human newborns, infants, and young children. Pediatrics 6:467-472

7. Bevilacqua MP, Nelson RM 1993 Selectins. J Clin Invest 91:379-387

8. Kishimoto TK, Jutila MA, Berg EL, Butcher EC 1989 Neutrophil Mac-1 and MEL-14 adhesion proteins inversely regulated by chemotactic factors. Science $245: 1238-1241$

9. Jutila MA, Rott L, Berg EL, Butcher EC 1989 Function and regulation of the neutrophil MEL-14 antigen in vivo: comparison with LFA-1 and Mac-1. J Immunol 143:3318-3324

10. Palecanda A, Walcheck B, Bishop DK, Jutila MA 1992 Rapid activation-independent shedding of leukocyte L-selectin induced by cross-linking of the surface antigen. Eur J Immunol 22:1279-1286

11. Anderson DC, Schmalstieg FC, Kohl S, Arnaout MA, Hughes BJ, Tosi MF, Buffone GJ, Brinkley BR, Dickey WD, Abramson JS, Springer TA, Boxer LA, Hollers JM, Smith CW 1984 Abnormalities of polymorphonuclear leukocyte function associated with a heritable deficiency of a high molecular weight surface glycoprotein (GP138): common relationship to diminished cell adherence. J Clin Invest 74:536-551

12. Kishimoto T, Larson RS, Corbi AL, Dustin ML, Staunton DE, Springer TA 1989 Leukocyte integrins. In: Springer TA, Anderson DC, Rothlein R, Rosenthal AS, eds. Structure and Function of Molecules Involved in Leukocyte Adhesion. SpringerVerlag, New York, pp 7-43

13. Jones DH, Schmalstieg FC, Dempsey K, Krater SS, Nannen DD, Smith CW, Anderson DC 1990 Subcellular distribution and mobilization of Mac-1 (CD11b/ CD18) in neonatal neutrophils. Blood 75:488-498

14. Vedder NB, Harlan JM 1988 Increased surface expression of CD11b/CD18 (Mac-1) is not required for stimulated neutrophil adherence to cultured endothelium. J Clin Invest 81:676-682

15. Rebuck N, Gibson A, Finn A 1995 Neutrophil adhesion molecules in term and premature infants; normal or enhanced leucocyte integrins but defective L-selectin expression and shedding. Clin Exp Immunol 101:183-189

16. Buhrer C, Graulich J, Dietger S, Dudenhausen JW, Obladen M 1994 L-Selectin is down-regulated in umbilical cord blood granulocytes and monocytes of newborn infants with acute bacterial infection. Pediatr Res 36:799-804

17. Smith JB, Kunjummen RD, Raghevender BH 1991 Eosinophils and neutrophils of human neonates have similar impairments of quantitative upregulation of Mac-1 (CD11b/CD18) expression in vitro. Pediatr Res 30:355-361

18. Anderson DC, Rothlein R, Marlin SD, Krater SS, Smith CW 1990 Impaired transendothelial migration by neonatal neutrophils: abnormalities of Mac-1 (CD11b/ CD18)-dependent adherence reactions. Blood 76:2613-2621

19. Bruce MC, Baley JE, Medvik KA, Berger M 1987 Impaired surface membrane expression of $\mathrm{C} 3 \mathrm{bi}$ but not $\mathrm{C} 3 \mathrm{~b}$ receptors on neonatal neutrophils. Pediatr Res 21:306-311

20. Schleiffenbaum B, Spertini O, Tedder TF 1992 Soluble L-selectin is present in human plasma at high levels and retains functional activity. J Cell Biol 119:229-238

21. Koenig JM, Jutta S, Anderson DC, Smith EO, Smith CW 1996 Diminished soluble and total cellular L-selectin in cord blood is associated with its impaired shedding from activated neutrophils. Pediatr Res 39:616-621

22. Fearon DT, Collins LA 1983 Increased expression of C3b receptors on polymorphonuclear leukocytes induced by chemotactic factors and by purification procedures. J Immunol 130:370-375

23. Smith JB, Tabsh KMA 1993 Fetal neutrophils and eosinophils express normal levels of L-selectin. Pediatr Res 34:253-257

24. Mulligan MS, Vaporciyan AA, Warner RL, Jones ML, Foreman KE, Miyasaka M, Todd RF, Ward PA 1995 Compartmentalized roles for leukocytic adhesion molecules in lung inflammatory injury. J Immunol 154:1350-1363

25. Fortenberry JD, Marolda JR, Anderson DC, Smith CW, Mariscalco MM 1994 CD-18-dependent and L-selectin-dependent neutrophil emigration is diminished in neonatal rabbits. Blood 84:889-897

26. Waisman D, VanEeden SF, Hogg JC, Solimano A, Massing B, Bondy GP 1998 L-Selectin expression on polymorphonuclear leukocytes and monocytes in premature infants: reduced expression after dexamethasone treatment for bronchopulmonary dysplasia. J Pediatr 132:53-56

27. Buhrer C, Stibenz D, Graulich J, Gernhold U, Butcher EC, Dudenhausen JW, Obladen M 1995 Soluble L-selectin (sCD62L) umbilical cord plasma levels increase with gestational age. Pediatr Res 38:336-341

28. Erdman SH, Christensen RD, Bradley PP, Rothstein G 1982 The supply and release of storage neutrophils: a developmental study. Biol Neonate 41:132-136

29. Weinschenk NP, Farina A, Bianchi DW 2000 Premature infants respond to earlyonset and late-onset sepsis with leukocyte activation. J Pediatr 137:345-350

30. Weirich E, Rabin RL, Maldonado Y, Benitz W, Modler S, Herzenberg LA 1998 Neutrophil CD11b expression as a diagnostic marker for early-onset neonatal infection. J Pediatr 132:445-451

31. Fortenberry JD, Bhardwaj V, Niemer P, Cornish JD, Wright JA, Bland L 1996 Neutrophil and cytokine activation with neonatal extracorporeal membrane oxygenation. J Pediatr 128:670-678

32. Weinschenk NP, Farina A, Bianchi DW 1998 Neonatal neutrophil activation is a function of labor length in preterm infants. Pediatr Res 44:942-945

33. Usmani SS, Kamran S, Harper RG, Wapnir RA, Mehta R 1993 Effect of maternal labor and mode of delivery on polymorphonuclear leukocyte function in healthy neonates. Pediatr Res 33:466-468 